University of Nebraska - Lincoln DigitalCommons@University of Nebraska - Lincoln

CSE Conference and Workshop Papers

Computer Science and Engineering, Department of

2017

\title{
Smart Underground Antenna Arrays: A Soil Moisture Adaptive Beamforming Approach
}

\author{
Abdul Salam \\ salama@purdue.edu \\ Mehmet C. Vuran \\ University of Nebraska-Lincoln, mcvuran@cse.unl.edu
}

Follow this and additional works at: http://digitalcommons.unl.edu/cseconfwork

Part of the Computer Engineering Commons, Electrical and Computer Engineering Commons, and the Other Computer Sciences Commons

Salam, Abdul and Vuran, Mehmet C., "Smart Underground Antenna Arrays: A Soil Moisture Adaptive Beamforming Approach" (2017). CSE Conference and Workshop Papers. 306.

http://digitalcommons.unl.edu/cseconfwork/306

This Article is brought to you for free and open access by the Computer Science and Engineering, Department of at DigitalCommons@University of Nebraska - Lincoln. It has been accepted for inclusion in CSE Conference and Workshop Papers by an authorized administrator of

DigitalCommons@University of Nebraska - Lincoln. 


\title{
Smart Underground Antenna Arrays: A Soil Moisture Adaptive Beamforming Approach
}

\author{
Abdul Salam Mehmet C. Vuran \\ Cyber-Physical Networking Laboratory \\ Department of Computer Science \& Engineering \\ University of Nebraska-Lincoln, Lincoln, NE 68588 \\ Email: \{asalam, mcvuran\}@cse.unl.edu
}

\begin{abstract}
Current wireless underground (UG) communication techniques are limited by their achievable distance. In this paper, a novel framework for underground beamforming using adaptive antenna arrays is presented to extend communication distances for practical applications. Based on the analysis of propagation in wireless underground channel, a theoretical model is developed which uses soil moisture information to improve wireless underground communications performance. Array element in soil is analyzed empirically and impacts of soil type and soil moisture on return loss (RL) and resonant frequency are investigated. Accordingly, beam patterns are analyzed to communicate with underground and above ground devices. Depending on the incident angle, refraction from soil-air interface has adverse effects in the UG communications. It is shown that beam steering improves UG communications by providing a high-gain lateral wave. To this end, the angle, which enhances lateral wave, is shown to be a function of dielectric properties of the soil, soil moisture, and soil texture. Evaluations show that this critical angle varies from $0^{\circ}$ to $16^{\circ}$ and decreases with soil moisture. Accordingly, a soil moisture adaptive beamforming (SMABF) algorithm is developed for planar array structures and evaluated with different optimization approaches to improve UG communication performance.
\end{abstract}

\section{INTRODUCTION}

Despite the recent developments in wireless underground (UG) communications [2], the communication ranges are still limited for many potential applications. Therefore, advanced techniques, which are designed based on the unique characteristics of the wireless UG channel, are required to extend the communication ranges. Soil properties and soil moisture significantly impact the UG communications [25]. This necessitates the adaption of parameters of the UG communication system based on the changing environment. Such adaption requires tight integration of soil sensing technologies with the communication devices to improve UG communication performance. For an UG antenna, change in soil moisture requires changing operation frequency and bandwidth to maintain high throughput and gain [6]. Similarly, to enhance UG communications ranges, maximum energy should be focused at a particular angle which should be determined dynamically according to ambient soil properties [25]. Due to these phenomena, the use of a high-gain fixed-directional antennas [29], which lack the capability to adjust their beam direction dynamically, may not result in ideal system performance. To this end, a soil moisture adaptive beamforming (SMABF) approach, based on antenna arrays, is developed in this paper. SMBAF adjusts its parameters and beams the maximum energy at the desired angle based on ambient environmental conditions.

Many factors impact beamforming from UG antenna arrays. The distance that waves travel from each antenna element to reach the soil-air interface is different based on the array geometry. Change in index of refraction causes delay in the speed of beams. Soil moisture variations lead to change in the resonant frequency of antenna elements. Bandwidth, return loss (RL), and reflection coefficients at the resonant frequency also change with soil moisture. Moreover, a reliable beamforming architecture requires deep understanding of the propagation in the wireless communication channel to exploit the nature of spatial properties of multipath components for an effective beamforming solution.

In addition to physical constraints, from a networking perspective, communication from an UG node to another UG node (UG2UG) and an aboveground node (UG2AG) require different beam shapes. In UG2UG communications, lateral wave is the most dominant component and travels along the soil-air interface [25], [31]. The lateral component has the potential, due to its lower attenuation, to reach higher distances. This lateral wave is formed and carries the most energy when antenna orientation is at a specific angle. This angle varies with variations in soil moisture and also depends on soil properties such as soil texture, and bulk density. On the contrary, for UG2AG communication, energy needs to be focused in the broadside to avoid refraction losses at the soil-air interface. Differences in wave propagation in these two links require different angles at which waves should be incident at soil-air interface. Due to these factors, adjustment of the phase at the UG antenna elements need phase alignment to add up coherently to avoid errors in beam steering.

To the best of our knowledge, this is the first work to propose soil moisture adaptive beamforming (SMABF) for UG communications. Antenna array structures buried underground are considered which communicate through soil and air. We analyze the UG channel impulse response model from a UG beamforming perspective. Challenges in UG beamforming are highlighted and use of soil adaptive beamforming approach is motivated. We present the effects of different soil properties on single antenna array element. The proposed mechanism 

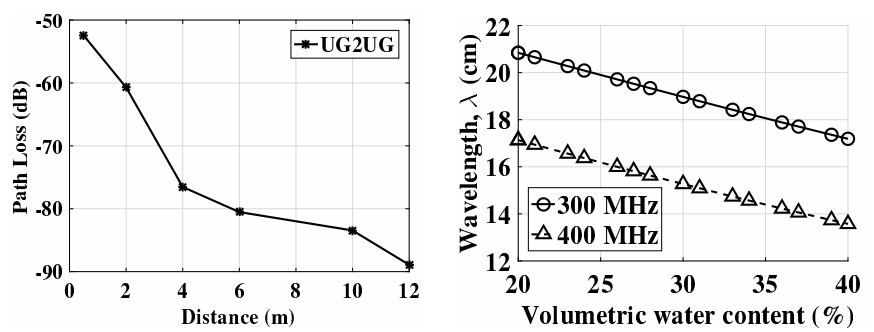

(b)

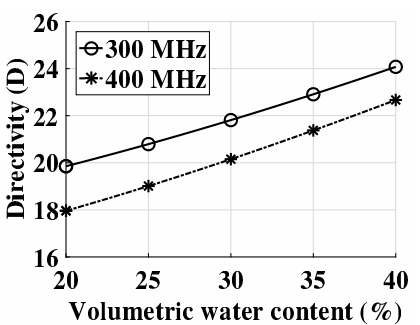

(c)

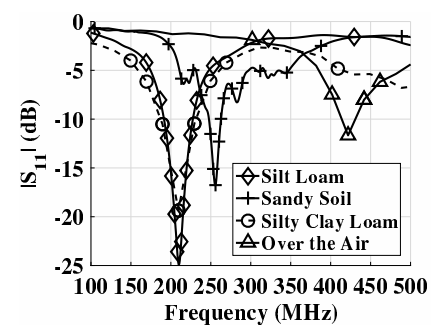

(d)

Fig. 1: (a) Pathloss in UG2UG channel, (b) Change in wavelength with change in soil moisture (c) Array directivity with change in soil moisture (d) Reflection coefficients of a dipole array element.

estimates the best beam steering angle based on the soil moisture sensing. Next, based on the optimal angle, a steering algorithm is developed for beamforming. This method works on array element weighting based on the UG2UG and UG2AG communications. Array element positions, inter-element distance are analyzed for best performance. Then an optimization algorithm is developed which is based on soil moisture sensing information. Sidelobe reduction is accomplished by using element thinning, and element positions optimization. Performance analysis based on testbed experiments and simulation results of SMABF communications are presented.

The rest of the paper is organized as follows: the related work is discussed in Section II. The channel model is discussed in Section III. Challenges to UG beamforming are presented in Section IV. An antenna array element in soil is analyzed in Section V. Design of SMABF array and steering algorithm is given in Section VI. Results are presented in Section VII. We conclude in Section VIII.

\section{RELATED WORK}

Wireless UG channel is the medium of communication in Internet of Underground Things (IOUT) [22], [24], which are being used in the area of precision agriculture [2], [4], [5], [8], [12], [22], [29], border monitoring [3], land slide monitoring, and pipeline monitoring [12], [27]. IOUT communications are based on both EM-wave propagation [31] and magnetic induction (MI) communications [28]. IOUT include MI, use EM communication devices and sensors, partly or completely buried underground for real-time soil sensing and monitoring.

Beamforming antennas [19] are used in wireless networks to reduce interference and improve capacity. Beamforming has been studied in [18] for over-the-air (OTA) wireless channels and in [17], for MI power transfer. However, MI beamforming cannot be readily applied to IOUT because the spatial multipath modularity does not exist in MI, and sender-receiver coils have to be parallel to each other in MIcommunications, which is a restriction which can be avoided in UG communications. In UG communications, lateral component [16] has the potential, via beam-forming techniques, to reach farther UG distances, which otherwise are limited $(8 \mathrm{~m}$ to $12 \mathrm{~m})$ because of higher attenuation in soil [25]. To the best of our knowledge, adaptive UG beamforming has not been studied before in literature, and this is the first work to develop soil moisture adaptive UG beamforming for the wireless UG channel.

\section{Channel MOdEl FOR SMABF}

Estimation of propagation characteristics through the soil is crucial to design a UG communication systems. The UG channel impulse response can be expressed as a sum of direct, reflected and lateral waves [25]:

$h_{u g}(t)=\sum_{l=0}^{L-1} \alpha_{l} \delta\left(t-\tau_{l}\right)+\sum_{s=0}^{S-1} \alpha_{s} \delta\left(t-\tau_{s}\right)+\sum_{r=0}^{R-1} \alpha_{r} \delta\left(t-\tau_{r}\right)$

where $L, S$, and $R$ are the number of multipaths; $\alpha_{l}, \alpha_{s}$, and $\alpha_{r}$ are complex gains; and $\tau_{l}, \tau_{s}$, and $\tau_{r}$ are delays associated with lateral wave, direct wave, and reflected wave, respectively. We highlight in [25] that based on the power delay profile (PDP) of wireless UG channel, lateral wave is the strongest component because it suffers from lower attenuation as it propagates through the air along the soil-air interface. Reflected and direct waves undergo higher attenuation due to the higher losses in soil medium. This unique phenomenon allows the design of buried antenna arrays with reasonable sizes. Furthermore, because of the unique three-wave structure of the PDP by focusing the transmitted energy to lateral waves, the delay spread can be further decreased, leading to higher data rates and long-range communications. It is worth noting that the interactions between soil, antennas, and the UG channel create unique opportunities that are not possible in other media. Moreover, due to higher permittivity of soil as compared to air, wavelength at a particular frequency is lower than that of air. This allows the use of lower frequency waves, which attenuate less in soil, with smaller-size antennas.

In Fig. 1(a), attenuation with distance is shown. Channel transfer functions are measured for dipole antennas buried at $20 \mathrm{~cm}$ depth up to the distance of $12 \mathrm{~m}$. A $30 \mathrm{~dB}$ path loss is observed when UG distance increases from $2 \mathrm{~m}$ to $12 \mathrm{~m}$. Due to these factors, an impedance-matched antenna for OTA communication is not matched in soil and new designs are necessary [8]. High attenuation in soil is one of the limiting factors for long range communications.

Due to their buried deployment and the dominance of the lateral wave in the wireless UG channel, sending signals in an isotropic direction (i.e., partly towards the Earth) would be waste of the resources. Thus, SMABF aims to communicate 


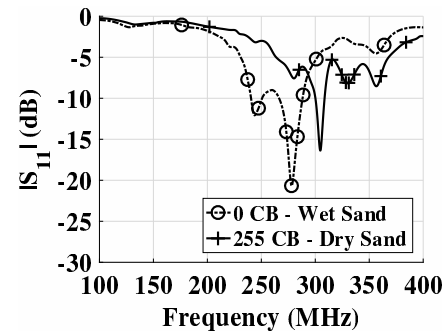

(a)

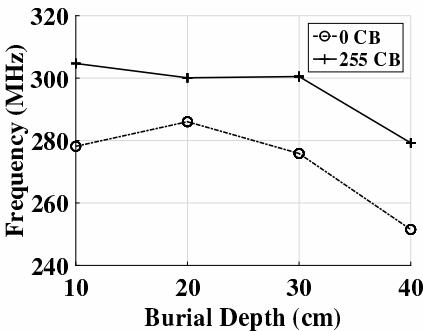

(b)

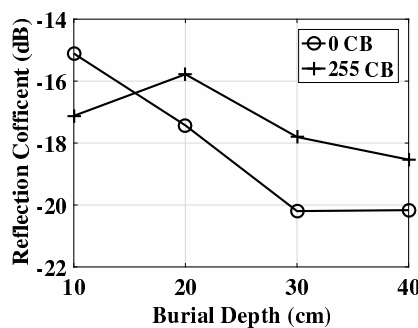

(c)

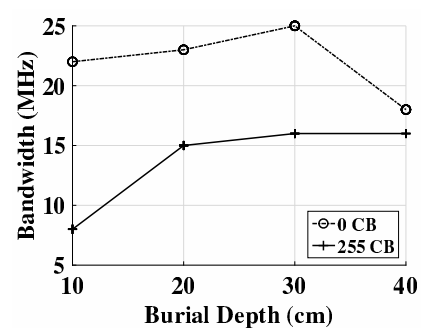

(d)

Fig. 2: Return loss in sandy soil, : (a) $S_{11}$ at different frequencies, (b) Change in resonant frequency with burial depth, (c) Reflection coefficient (dB) at different burial depths, (d) Antenna bandwidth at different burial depths for near-saturation and dry sandy soil.

with UG and AG devices by forming a focused narrow width beam in the desired direction, hence, extending the communication ranges.

\section{Challenges in Underground Beamforming}

In this section, we first analyze a fixed-beam system based on impacts of soil moisture on wavelength and directivity. Accordingly, we review UG beamforming challenges.

Impact of Soil Moisture on Wavelength: Wavelength in soil is calculated as $\lambda_{s}=(2 \pi) / k_{s}$, where $k_{s}$ is the wave number in soil ([23, Appendix B]). In Fig. 1(b), change in wavelength is shown as a function of volumetric water content (VWC). It can be observed that when VWC increases from $20 \%$ to $40 \%$, wavelength at $300 \mathrm{MHz}$ decreases from $21 \mathrm{~cm}$ to $17 \mathrm{~cm}$. Similarly at $400 \mathrm{MHz}$ wavelength decreases from $17 \mathrm{~cm}$ to $14 \mathrm{~cm}$. Accordingly, for an antenna array, the distance between succeeding elements needs to be selected in a way to accommodate wavelength changes due to soil moisture variations without affecting the directivity and beam patterns.

Impact of Soil Moisture on Directivity: Directivity of an one-dimensional UG antenna array can be expressed as $D \approx 2 \frac{N d}{\lambda_{s}}[13]$, where $N$ is the number of elements, $d$ is the distance between elements, and $\lambda_{s}$ is the wavelength in soil. In Fig. 1(c), directivity pattern is shown with change in soil moisture for antenna elements that are half wavelength $\lambda_{0} / 2$ (in the air) apart. It can be observed that soil moisture leads to linear changes in directivity, which needs to be mitigated for SMABF. Moreover, since the UG communication devices are buried to the close proximity of soil-air interface in homogeneous soil [8], therefore, soil moisture changes are not abrupt. Analysis of the layered soil effects on UG communications is left for future investigation. Phased arrays are used to steer the main beam of the antenna without physically moving the antenna [9], [11], [15]. Due to the requirement of accurate phase control with wavelength change, smart antennas with phase shifters are suitable in UG communications. In the following, we analyze the effects of soil on UG beamforming, and design a UG SMABF solution which is robust and adaptive to these variations.

\section{Analysis of Single ARray Element in SoIL}

We first analyze the behavior of a single array element in soil medium. To this end, first, an antenna element in soil is compared with an OTA antenna element through empirical evaluations in an indoor testbed [25]. The indoor testbed provides flexible control over the soil moisture, and holds dipole antennas at $10 \mathrm{~cm}, 20 \mathrm{~cm}, 30 \mathrm{~cm}$, and $40 \mathrm{~cm}$ depths for transmitter-receiver distances of $50 \mathrm{~cm}$, and $1 \mathrm{~m}$. Then, the array element impedance and soil-air interface effects are analyzed by taking RL measurements.

\section{A. Comparison of In-Soil and OTA Array Element}

Return Loss in Soil: In Fig. 1(d), the performance of single array element (dipole) buried in soil is compared with that of a free space element. Return loss measurements of a $433 \mathrm{MHz}$ OTA antenna element in three different soils are shown for a frequency range of $100 \mathrm{MHz}$ to $500 \mathrm{MHz}$. It can be observed that the resonant frequency of the antenna shifts to lower frequency values when buried underground. Resonant frequency in silt loam soil is $202 \mathrm{MHz}$, in silty clay loam (SCL) it is $209 \mathrm{MHz}$, and in sandy soil resonant frequency is $278 \mathrm{MHz}$. Resonant frequency in sandy soil is $76 \mathrm{MHz}$ higher than the silt loam soil. This is because the relative permittivity of a particular soil depends on its net water content [21] and silt loam has a higher water holding capacity than sandy soil. Therefore, due to silt loam's higher relative permittivity, lower resonant frequency is observed. Next, we analyze the effects of soil moisture variations on the RL, and resonant frequency of the array element.

Impact of Soil Moisture on Element RL: In Fig. 2(a), RL of element in silt loam at $10 \mathrm{~cm}$ depth is shown for soil matric potential values of 0 and $255 \mathrm{CB}$. When soil moisture decreases (matric potential changes from 0 to $255 \mathrm{CB}$ ), resonant frequency has increased from $278 \mathrm{MHz}$ to $305 \mathrm{MHz}$. Effects of change in soil moisture on the resonant frequency at different depths are shown in Fig. 2(b). At $20 \mathrm{~cm}$, with change in soil moisture from 0 to $255 \mathrm{CB}$, resonant frequency increases from $276 \mathrm{MHz}$ to $301 \mathrm{MHz}$. With the similar change at $30 \mathrm{~cm}$ depth, resonant frequency changes from $276 \mathrm{MHz}$ to $301 \mathrm{MHz}$, and at $40 \mathrm{~cm}$ depth, it changes from 251 to $279 \mathrm{MHz}$. Analysis of the RL of antenna (Fig. 2(a)-2(d)) in sandy soil at different burial depths and soil moisture levels shows that the RL of the antenna changes with the soil moisture. Resonant frequency moves to lower frequency ranges when the soil moisture increases. Moreover, unlike OTA communications, the optimal frequency where the maximum capacity is achieved is not the same as the resonant frequency of the antenna [6]. 


\section{B. Element Impedance in Soil}

The knowledge of impedance of an array element in soil is important to match the array to a transmission line. For efficient wireless communication, the impedance of an antenna element, $Z_{a}$, should be matched to the output impedance of the transceiver, $Z_{s}$, such that the radiated power is maximized and the returned power to the transmitter is minimized. Due to soil-air interface effects, soil cannot be considered as an infinite medium, as is typically considered in OTA antenna models. Consequently, antenna RL is not merely a shift in spectrum space when the antenna is moved from air to soil, but the shape of the RL curve also changes.

Soil-Air Interface Impacts on Element Impedance: When a buried antenna is excited, a current distribution of $I_{0}(\zeta)$ is generated along the antenna. The generated wave propagates towards the soil-air interface, where it is reflected and refracted. The reflected electric field, $E_{r}$, that reaches the antenna induces an additional current, $I_{r}$, on the antenna, affecting its impedance [30]. The induced current further impacts the generated wave and higher order reflection effects exist. However, due to the high attenuation in soil, these higher order effects are negligible and only the first order effects are considered. The induced current on the dipole, $I_{r}$, as well as the resulting impedance, $Z_{r}$, can be modeled as the result of a field generated by an imaginary dipole placed in a homogeneous soil environment. Accordingly, $Z_{r}$ is modeled based on a modified mutual impedance model between two dipole antennas [20] and the reflection coefficient at the soilair interface. The mutual impedance, $Z_{r}$, is then added to the self impedance, $Z_{a}$, to obtain the total impedance of the buried antenna in half space [30]. With insights gained from the analysis of individual antenna element, we design multielement SMABF array next.

\section{DESIGN OF SMABF ARRAY}

In this section, we investigate array configuration and element positioning of phased array antenna for UG communications (Section VI-A). In Section VI-B, beam patterns for UG2AG communications are developed. UG2UG beam patterns are analyzed in Section VI-C. In this development, we emphasize the beamforming aspects related to the UG2UG and UG2AG communications without going into details of beamforming basics. For a comprehensive treatment of the subject, we refer the reader to [10].

\section{A. Array Layout and Element Positioning}

First, we investigate the desired size and number of antenna elements in the SMABF array which can form beams to communicate with $\mathrm{UG}$ and $\mathrm{AG}$ devices. The AG nodes can be fixed

TABLE I: UG2UG and UG2AG steering angles.

\begin{tabular}{|c|c|c|c|}
\hline \multicolumn{2}{|c|}{ Communication Link } & $\boldsymbol{\theta}$ & $\boldsymbol{\phi}$ \\
\hline \multirow{2}{*}{ UG2AG } & No Steering & $0^{\circ}$ & $0^{\circ}$ \\
\cline { 2 - 4 } & Beam Steering & $0^{\circ}-60^{\circ}$ & $0^{\circ}$ \\
\hline \multirow{3}{*}{ UG2UG } & Lateral Wave & $\begin{array}{c}\text { VWC Dependent } \\
\text { (Sect. VI-C) }\end{array}$ & $0^{\circ}$ \\
\cline { 2 - 4 } & Direct Wave - X Orientation & $90^{\circ}$ & $0^{\circ}$ \\
\cline { 2 - 4 } & Direct Wave - Y Orientation & $90^{\circ}$ & $90^{\circ}$ \\
\hline
\end{tabular}

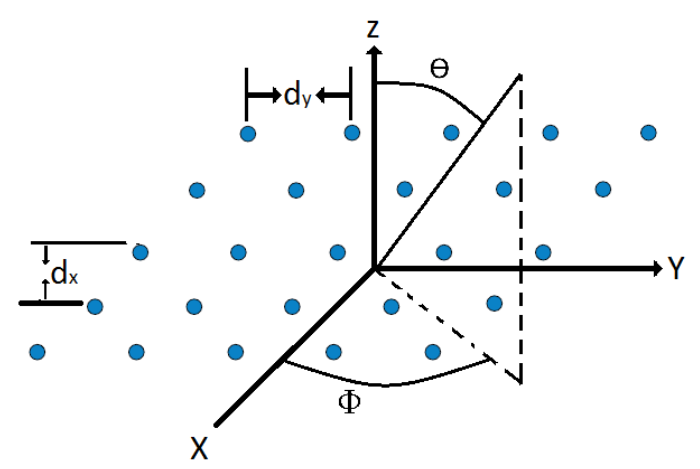

Fig. 3: Arrangement of array elements in a planar grid.

sinks or mobile nodes mounted on movable infrastructures. Then, we analyze SMABF inter-element spacing.

Following features are desirable in the design an SMABF antenna array: 1) Due to wavelength changes in soil, interelement spacing should be such that the directivity and desired beam shape are not lost significantly with changes in soil conditions, 2) Array is to be designed to work in a wide range of frequencies, 3) Elements are half-wave length with support for multiple inter-element spacing, 4) The array is to have number of elements which are not prohibitive for UG deployment and maintains higher directivity, 5) Both UG2UG and UG2AG array patterns are desirable with support of steering angles, 6) It should be able to adjust its parameters when the soil moisture changes.

\section{B. UG2AG Communication Beam Pattern}

Since UG2AG link is different than the UG2UG link, energy radiated in the vertical direction from the buried SMABF array needs to be determined at different receiver angles. Experiments conducted for a UG sender buried at a depth of $20 \mathrm{~cm}$ to an AG node at different distances and angles in [25] show that for the receiver at the angle of $0^{\circ}$, highest attenuation occurs, whereas the lowest attenuation is observed at $90^{\circ}$. At $90^{\circ}$, the wave does not experience high refraction compared to the $0^{\circ}$ case. Therefore, in UG2AG communications, the wave energy directed closer to the normal of the soil surface leads to higher gains and throughput. In the following, we discuss two scenarios of UG2AG communications.

Case 1: Beam Steering. We consider an $M \times N$ planar array where the array elements are arranged in a twodimensional rectangular grid, with inter-element spacing $d_{x}$, and $d_{y}$ in the $x$ and $y$ directions, respectively (Fig. 3). For the reminder of the paper, we assume that $d_{x}=d_{y}$. If the precise location of the AG node $\left(\theta_{A G}, \phi_{A G}\right)$ is known (i.e. through GPS), then beam is steered accordingly by adding the phase shifts $\delta_{i j}$ at the $i j^{\text {th }}$ element. Accordingly, the array factor for UG2AG pattern can be expressed as [15]:

$$
\begin{gathered}
A F_{b s}(\theta, \phi)=\sum_{i=1}^{M} \sum_{j=1}^{N} w_{i j} \exp \left(-\left[j k _ { s } \left(x_{i j} \sin \theta \cos \phi\right.\right.\right. \\
\left.\left.\left.+y_{i j} \sin \theta \sin \phi\right)\right]+\delta_{i j}\right)
\end{gathered}
$$


where $k_{s}$ is the wave number in soil ([23, Appendix B]), and for the $i j^{t h}$ antenna element, $w_{i j}$ is the weight, $x_{i j}$ and $y_{i j}$ are the coordinates, and $\delta_{i j}$ is the phase shift. For the intended direction $\left(\theta_{A G}, \phi_{A G}\right)$, the phase shift is [9]:

$$
\delta_{i j}=-k_{s}\left(x_{i j} \sin \theta_{A G} \cos \phi_{A G}+y_{i j} \sin \theta_{A G} \sin \phi_{A G}\right) \forall i, j .
$$

It can be observed from (2-3) that both the array factor and the antenna element phase shifts are a functions of wave number in soil, $k_{s}$, which is a function of soil moisture. Therefore, compared to OTA beamforming, for UG beam steering, the antenna element phase shifts need to be dynamically adjusted to maintain beams formed at a particular direction when soil moisture changes.

Case 2: Refraction Adjustment. When UG2AG beam is steered at angles other than normal to the soil-air interface, RF waves experience refraction. The refraction process not only degrades the performance of the SMABF but also changes the angle-of-arrival at the AG nodes. Moreover, an optimal angle of incidence exists with respect to burial depth of the SMABF antenna array, at which refraction is more dominant. Hence, less reflection of incidence wave occurs. Moreover, these phenomena result in different propagation speeds because of different refraction indices of soil and air, leading to spreading, and decay of focused beam. Due to these factors, adjustment of the phase at the UG antenna elements does not align the phase to add up coherently and leads to errors in beam steering and beam pointing direction. Depending on the incident angle, this has adverse effects in the UG communications. The error caused by refraction from soil-air interface is called beam squint [15] and results in time dispersion of the signal.

To address this issue, we use time-delay beam steering [15] in SMABF to align signal envelopes and achieve the desired performance to mitigate soil-air interface effects. Time delay units are used to adjust the beam pointing direction by using the refraction angle. Given the position of the AG node, $\left(\theta_{A G}, \phi_{A G}\right)$, time delay to correct this effect, $\tau_{i j}$, is expressed as [14]:

$$
\tau_{i j}=\sin \theta_{r} \times h\left[i \times d_{x} \cos \phi_{r}+j \times d_{y} \sin \phi_{r}\right] / S, \quad \forall i, j,
$$

where $S$ is the speed of the wave in soil ([23, Appendix C]), $d_{x}$ and $d_{y}$ are the element spacing in the $x$ and $y$ direction respectively, $h$ is the burial depth, and $\theta_{r}$ is the refraction angle, which is calculated by Snell's law as:

$$
\theta_{r}=\arcsin \left(\frac{\eta_{a}}{\eta_{s}} \sin \theta_{A G}\right)
$$

where $\eta_{a}$, and $\eta_{s}$ are the refractive indices of air, and soil, respectively.

In (4), $\tau_{i j}$ is a function of burial depth from soil-air interface, and soil moisture. Higher refraction index (slow speed of wave in soil) leads to higher delay. Once $\tau$ and $\delta_{i}$ are determined, the array factor is expressed as [15]:

$$
\begin{aligned}
A F_{r a}(\theta, \phi)= & \sum_{i=1}^{M} \sum_{j=1}^{N} w_{i j} \exp \left(-\left[j k _ { s } \left(x_{i j} \sin \theta \cos \phi\right.\right.\right. \\
& \left.\left.+y_{i j} \sin \theta \sin \phi\right)+2 \pi f \tau_{i j}+\delta_{i j}\right],
\end{aligned}
$$

Next, we analyze the UG2UG communication beam pattern.

\section{UG2UG Communication Beam Pattern}

In this section, two scenarios for UG2UG communications are discussed. First, we investigate the optimal angle for soil moisture-based beam steering using lateral waves. Then, the case for direct wave communication is discussed.

Case - 1: Estimation of Soil Moisture-Based Optimum Steering Angle: It has been shown in [7], [25], that in UG communications lateral wave travels along the soil-air interface to reach the receiver. This lateral wave is maximized if the energy from the UG antenna is radiated in an optimum angle $\theta_{U G}^{*}$. This angle depends on the dielectric properties of the soil and is given by [26]:

$$
\theta_{U G}^{*}=\frac{1}{2} \tan ^{-1}\left(\frac{2 R e\left(\eta_{s}^{2}-1\right)^{1 / 2}}{\left|\eta_{s}^{2}-1\right|-1}\right) \mathrm{rad}
$$

where $\theta_{U G}^{*}$ is used to indicate optimum value, and $\eta_{s}$ is the refractive index of the soil. The derivation of optimal angle, $\theta_{U G}^{*}$, is given in ([23, Appendix A]).

Case - 2: Direct Wave. For short UG2UG communication distances, when direct wave is more dominant than the lateral wave, communication is enhanced by forming a direct UG beam towards the receiver UG node through the soil. Steering angles for lateral and direct wave beams are given in Table. I. In both cases, (2) is used based on the desired beam pattern.

\section{SMABF Directivity Maximization}

Consequently, directivity of a SMABF array is defined as [9]:

$$
D=\frac{4 \pi\left|A F_{\max }\right|^{2}}{\int_{0}^{2 \pi} \int_{0}^{\pi}|A F|^{2} \sin \theta d \theta d \phi},
$$

where $A F_{\max }$ is the main beam peak (maximum of the array factor).

In the UG channel, wavelength changes with soil moisture, hence, fixed inter-element spacing results in deterioration of array factor, and decreased directivity. This is unique to the UG channel, since in OTA channel wavelength remains fixed, hence, inter-element spacing does not change and directivity does not vary. Therefore, with soil moisture changes, the goal is to optimize the inter-element spacing which maximize directivity and avoids grating lobes. This optimization problem is formulated as [9]:

$$
\wp: \max D \quad \text { s.t. } \frac{d_{x}}{\lambda_{s}}<\frac{1}{1+\sin \theta_{U G}},
$$

where $D$ is directivity (8), $\theta_{U G}$ is the steering angle for UG2UG and UG2AG communications from the broadside, $d_{x}$ is the inter-element spacing in the $x$ and $y$ direction, respectively, and $\lambda_{s}$ is the wavelength in soil. 


\section{E. SMABF Element Thinning Through Virtual Arrays}

To maintain a optimum inter-element spacing, implementation of $d_{x}^{*}$ is not feasible from practical design point, therefore, SMABF uses array thinning (virtual arrays) [15], to adapt to wavelength changes due to soil moisture variations. In UG array thinning, a subset of the elements from the full planar structure is selected to avoid grating lobes. Through element thinning, virtual arrays of elements are formed, where the physical antenna elements are turned on and off. By using this approach, optimum configuration of elements is determined from the wavelength at the current soil moisture level. Virtual array inter-element spacing is denoted by $d_{x}^{v}$. Element weights $w_{i j}$ are turned on and off as following [15]:

$$
w_{i j}= \begin{cases}1 & \text { if } i \text { is multiple of } \frac{\left\lfloor d_{x}^{*}\right\rfloor}{d_{x}^{*}} \forall i, j \in K \\ 0 & \text { otherwise. }\end{cases}
$$

where $d_{x}^{*}$ is the optimal inter-element spacing, $i=j$, and $K$ is the total number of elements such that $K>M$. Virtual array inter-element spacing $d_{x}^{v}$ is chosen such that with change in wavelength due to soil moisture variations, higher directivity is maintained.

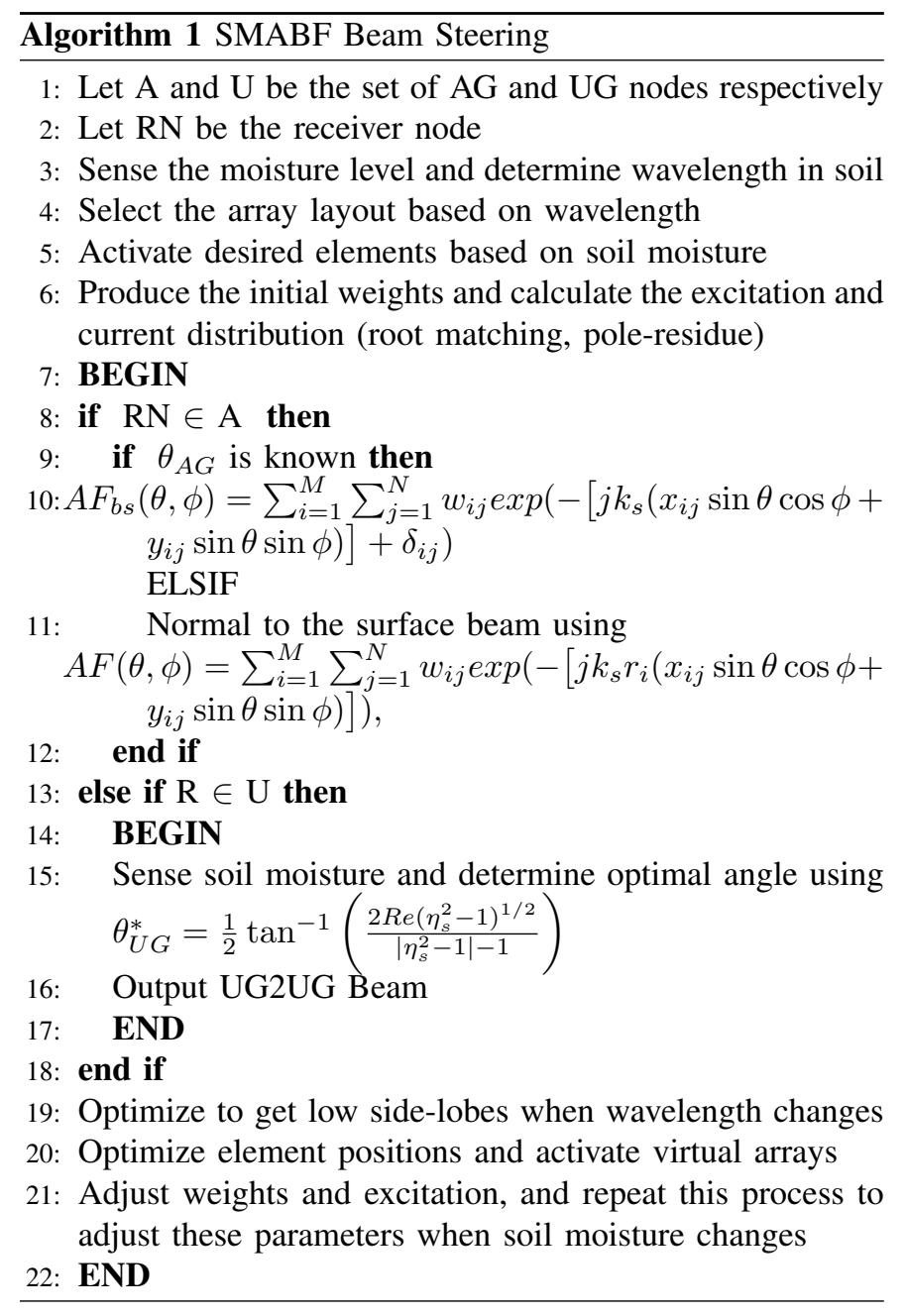

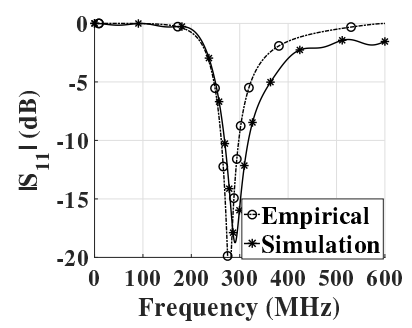

(a)

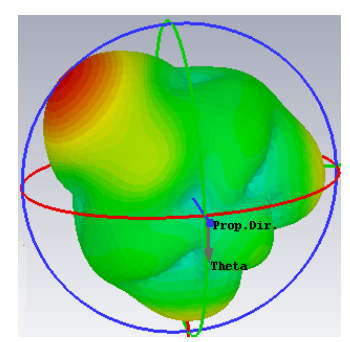

(b)
Fig. 4: (a) Comparison of measured and simulated reflection coefficients, (b) 3D view of UG2AG beam.

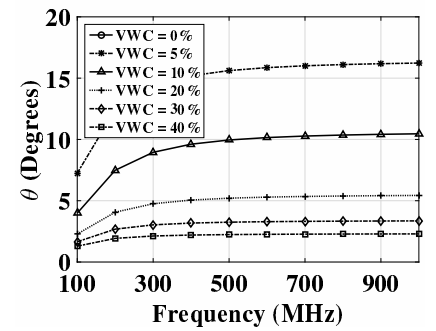

(a)

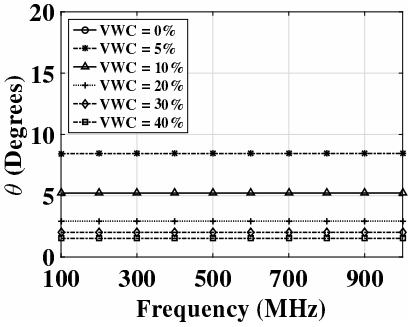

(b)
Fig. 5: Optimal angle with frequency in different soils: (a) Silty Clay Loam, (b) Sandy Soil.

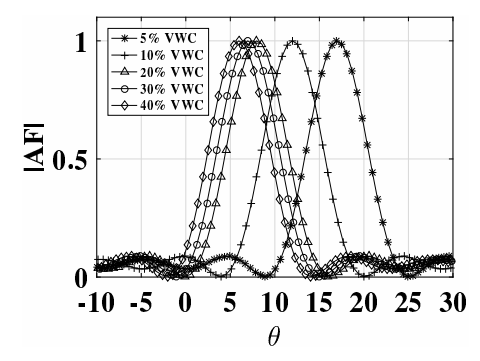

(a)

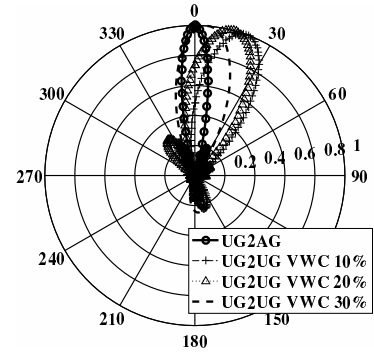

(b)
Fig. 6: (a) Array factor for UG2UG communications for different soil moisture levels, (b) UG2AG communications.

A beam steering algorithm is given in Algorithm 1 to produce different beam patterns required for UG2UG and UG2AG communications. This algorithm addresses the communication requirement on these two separate links.

\section{RESULTS}

In this section, first, SMABF simulation results are presented, then the developed model is validated through empiri$\mathrm{cal}$ and numerical evaluations. Then, comparisons of SMABF performance improvements with a nonadaptive system are presented.

SMABF Simulations: SMABF array design is evaluated through simulations in CST Microwave Studio (MWS), a simulation program which is used to simulate full wave 3D EM problems. A SMABF phased array antenna consisting of $5 \times 5$ dipole element has been simulated in sandy soil. Array is capable of operating in $0.2-0.6 \mathrm{GHz}$ in soil, and supports beam steering for communication links and angles given in Table I to maintain connectivity with UG and AG nodes.

First, a dipole antenna element is simulated in the sandy soil and different parameters are analyzed. Element is modeled using PEC cylinder material. Excitation is done using port 


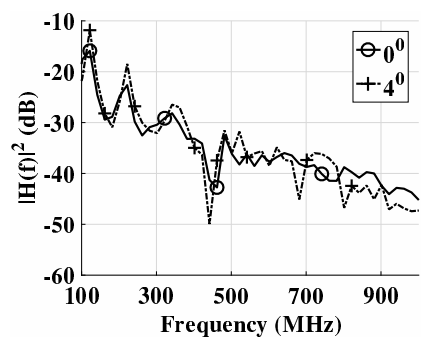

(a) Sandy $-50 \mathrm{~cm}$ T-R Distance

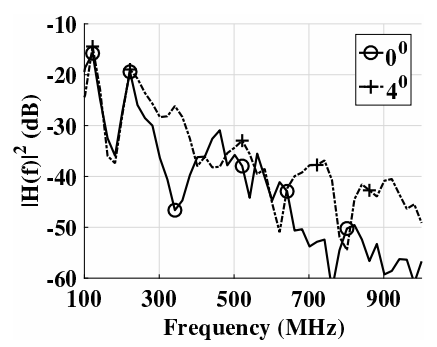

(b) Sandy - $1 \mathrm{~m}$ T-R Distance

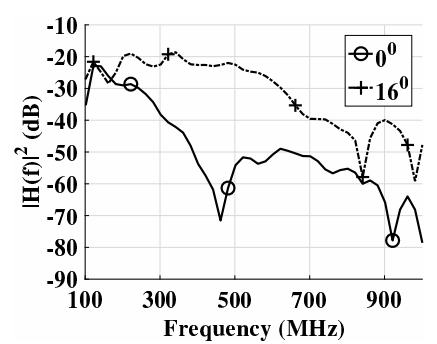

(c) SCL - $50 \mathrm{~cm}$ T-R Distance

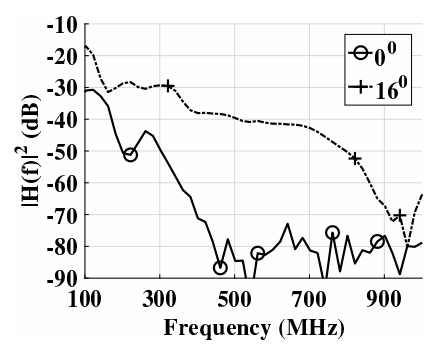

(d) SCL - $1 \mathrm{~m}$ T-R Distance

Fig. 7: Comparison of optimum angle UG communications with fixed orientation.

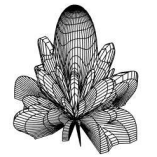

(a) $5 \%$

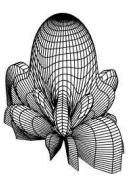

(f) $5 \%$

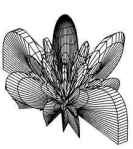

(b) $10 \%$

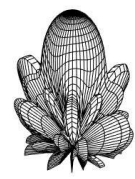

(g) $10 \%$

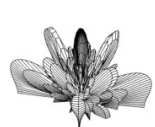

(c) $20 \%$

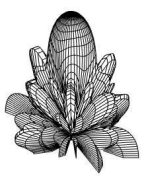

(h) $20 \%$

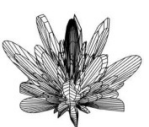

(d) $30 \%$

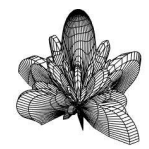

(i) $30 \%$

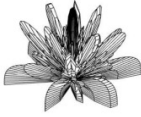

(e) $40 \%$

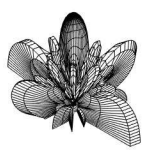

(j) $40 \%$

Fig. 8: Deterioration of array factor with change in soil moisture for a nonadaptive beamforming system. (a-e) SCL soil, (f-j) sandy soil.

placed in a gap in the middle of the element. OTA resonant frequency at one half-wavelength is $433 \mathrm{MHz}$. Higher mesh (40 per wavelength) is used for higher accuracy and timedomain solver is employed using unit cell approach. 50-ohms feed impedance is specified. S-parameters of the simulated element are compared with measurements to validate the simulated element design. Simulated and empirical results (Fig. 4(a)) show a very good agreement.

Once the individual SMABF element is simulated and validated, then a full array simulation configuration is created to incorporate element into the array design [1]. In CST MWS, once the UG2UG and UG2AG beam patterns are specified, a distribution matrix is calculated. This distribution matrix is used for element excitation to generate the desired beam pattern. With change in soil moisture, a new distribution matrix is produced to adjust the beam steering angle. A 3D view of UG2AG beam is shown in Fig. 4(b).

Optimum UG Angle: The optimum angle to maximize UG2UG lateral wave communication is obtained as a function of the properties of soil medium by using (7). We analyze the lateral wave angle for silty clay loam (SCL) and sandy soils for volumetric water content range of $0 \%$ to $40 \%$ in the frequency range of 100 to $1,000 \mathrm{MHz}$. Particle distributions of these two soils are shown in Table II.

In Fig. 5, optimal angle, $\theta_{U G}$, for different soils are shown as a function of frequency for soil moisture (VWC) range of

TABLE II: Particle Size Distribution and Classification of Testbed Soils [25].

\begin{tabular}{||c|c|c|c||}
\hline Textural Class & \%Sand & \%Silt & \% Clay \\
\hline Sandy Soil & 86 & 11 & 3 \\
\hline Silty Clay Loam & 13 & 55 & 32 \\
\hline
\end{tabular}

$0 \%$ to $40 \%$. It can be observed that optimal angle is higher in the SCL soil as compared to sandy soils. In SCL soil it goes up to $16^{\circ}$, whereas in sandy soil it is $9^{\circ}$. This is explained by the higher dielectric constant of the silty clay soil than that of the sandy soil. It can also be observed that optimal angle decreases with increase in soil moisture and it becomes close to zero when soil moisture (VWC) reaches to $40 \%$. This is attributed to increase in permittivity of soil due to increase in soil moisture. Summary of steering angles for $\mathrm{UG} 2 \mathrm{UG}$ and $\mathrm{UG} 2 \mathrm{AG}$ communications is given in Table I. UG beam patterns for different soil moisture levels are shown in Fig. 6(a)-6(b). In Fig. 6(a), linear plot of UG2UG array factor for different VWC values is shown. Polar plot with broadside UG2AG beam is shown in Fig.6(b). Next, enhancement in UG2UG communications are validated through empirical evaluations in SCL and sandy soil.

Empirical Evaluation of Lateral Wave Enhancement Through Optimum UG Angle: To evaluate the lateral wave enhancement, experiments are conducted in an indoor testbed in sandy soil, and in an outdoor testbed in silty clay soil [25]. By using a directional antenna buried at the $20 \mathrm{~cm}$ depth, measurements are conducted using a Keysight Fieldfox Vector Network Analyzer (VNA) N9923A. Chanel transfer functions are recorded and channel gain is determined, first, without the orientation change. Then, experiments are repeated by determining the optimum lateral wave angle and accordingly changing orientation in both soils. VWC values for sandy and SCL soil are $37 \%$ and $0 \%$, respectively, which lead to the optimum angle of $4^{\circ}$ in sandy soil and $16^{\circ}$ in SCL soil.

In Figs. 7, channel gain results of experiments conducted in SCL and sandy soils are shown for $50 \mathrm{~cm}$ and $1 \mathrm{~m}$ transmitter receiver (T-R) distance. It can be observed that, at $50 \mathrm{~cm} \mathrm{T-R}$ distance, when energy is directed at $4^{\circ}$ in sandy soil, a gain of $4 \mathrm{~dB}$ is realized at $500 \mathrm{MHz}$ as compared to no steering case (Fig. 7(a)). It can also be observed that by focusing energy in UG optimum angle, the channel gain is higher at higher

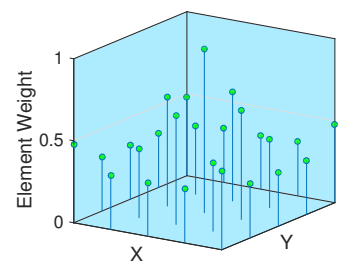

Fig. 9: Element weights for a $5 \times 5$ planar array in soil for broadside UG2AG pattern, for $40 \%$ soil moisture level, at $433 \mathrm{MHz}$. 


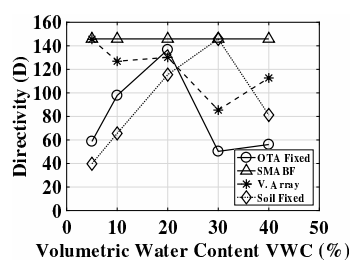

(a) SCL

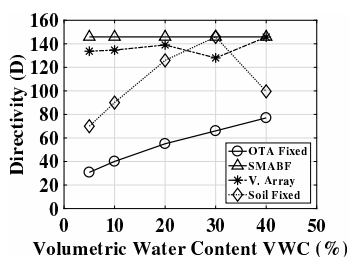

(b) Sand

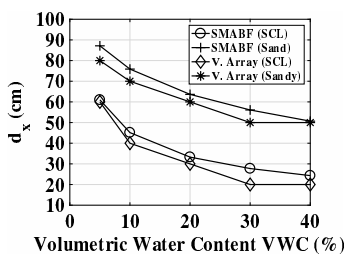

(c)

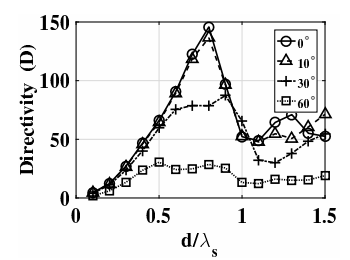

(d)

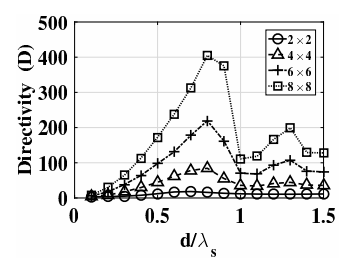

(e)

Fig. 10: (a) Change in directivity in change in soil moisture: (a) silty clay loam soil, (and) sandy soil, (c) inter-element spacing, $d_{x}$, to maximize directivity, (d) change in directivity with element spacing at different steering angles in sandy soil, (d) directivity with different array size in SCL soil.

frequencies, because path of the wave through soil is more affected by permittivity of the soil. In Fig. 7(b), channel gain in sandy soil for $1 \mathrm{~m} \mathrm{T-R} \mathrm{distance} \mathrm{is} \mathrm{shown.} \mathrm{It} \mathrm{can} \mathrm{be} \mathrm{observed}$ that at $1 \mathrm{~m}$, a $8 \mathrm{~dB}$ higher gain is achieved as compared to $50 \mathrm{~cm}$ because of the lower contribution by the direct wave at $0^{\circ}$ at $1 \mathrm{~m}$. Therefore, lateral wave communication is enhanced through optimum steering angle. An improvement of $32 \mathrm{~dB}$ and $37 \mathrm{~dB}$ channel gain is observed in SCL soil at $50 \mathrm{~cm}$ and $1 \mathrm{~m}$ distances, respectively, (Fig. 7(c) - Fig. 7(d)) as compared to fixed orientation. SCL soil has higher losses due to high permittivity of soil, which leads to high channel gains through UG lateral wave enhancement.

In the following sections, we first analyze the performance of a $5 \times 5$ SMABF planar array with an OTA fixed and a soil fixed system through numerical evaluations in MATLAB. These two fixed systems do not adapt their parameters to soil moisture variations. In OTA fixed system, in both soils, interelement spacing, $d_{x}$, is fixed at $433 \mathrm{MHz}$ OTA frequency halfwavelength, which is $34.64 \mathrm{~cm}$. In soil fixed scenario, the array deployment in both soils is customized for one particular soil moisture level (30\%). Accordingly, inter-element spacing for both soils is determined, and a fixed array design is deployed in soil without the support of the virtual arrays. For sandy soil, at $30 \%$ soil moisture level, $d_{x}=56 \mathrm{~cm}$, and in silty clay loam soil at $30 \%$ soil moisture level, $d_{x}=27 \mathrm{~cm}$. The halfwavelength inter-element spacing $d_{x}$ values, at $433 \mathrm{MHz}$, with $10 \%$ to $40 \%$ change in volumetric water content (VWC) are shown in Table III. Then, the performance of virtual arrays is analyzed. Virtual array inter-element spacing, $d_{x}^{v}$ is $10 \mathrm{~cm}$.

SMABF vs. Nonadaptive Beamforming: In this section, impacts of soil moisture variations on array factor and directivity are investigated. In Fig. 8, the deterioration of array factor with change in soil moisture for the OTA fixed beamforming system is shown in sandy and SCL soil, for the soil moisture (VWC) ranges from $5 \%$ to $40 \%$. In both soils, higher side lobes are observed when soil moisture increases from $5 \%$ to $40 \%$. However, in sandy soil, these effects of the change in soil moisture are less severe as compared to the silty clay soil. This is caused by larger wavelength changes due to soil moisture variations induced by higher permittivity in SCL soil. Element weights in soil for broadside UG2AG pattern, for $40 \%$ soil moisture level, at $433 \mathrm{MHz}$ are shown in Fig. 9.

Virtual Arrays: In virtual arrays, adaptive thinning is done based on wavelength changes due to soil moisture changes. Virtual SMABF array helps to maintain side-lobe levels and fixed directivity. It also avoids high side-lobe distortions as observed in nonadaptive beamforming case.

In Figs. 10(a)-10(b), directivity of SMABF and virtual array is compared with nonadaptive OTA fixed beamforming system for different soil moisture levels in sandy and SCL soils. Moreover, the change in directivity with change in soil moisture is also shown for the soil fixed deployment optimized at 30\% soil moisture level in both sandy and silt loam soil. It can be observed that the SMABF and virtual array system is able to adapt to soil moisture variations to maintain its directivity whereas drastic changes are observed in nonadaptive fixed OTA beamforming system in both soils. In sandy soil, at $5 \%$ soil moisture level, directivity is 115.09 less than the SMABF, and 75.67 less than the virtual array. When soil moisture increases to $40 \%$, directivity of nonadaptive system in sandy soil remains 68.81 less than the SMABF. Similarly, in SCL soil, for $10 \%$ and $30 \%$ soil moisture level, it is 48.05 and 95.57 below the optimum case. At $20 \%$ soil moisture level in fixed OTA SCL soil, directivity approaches close to the optimum case, which is caused by the resulting wavelength at $20 \%$ soil moisture becoming closer to $d_{x} / \lambda_{s}$, which leads to higher directivity.

It can also be observed from Figs. 10(a)-10(b), that in soil fixed nonadaptive system, for smaller changes in soil moisture (30\% to $20 \%$ ), directivity decrease is smaller (13\% decrease in sandy soil, and $21 \%$ decreases in SCL, when soil moisture decreases to $20 \%$ ). However, at 5\% soil moisture level, a 51\%, and $72 \%$ decrease is observed in sandy soil, and silty clay loam soil, respectively. Hence, in soils where soil moisture variations are not large (such as in growing crop soils), the deployment can be tailored to a recurrent soil moisture level to decrease complexity. Directivity in SMABF is maximized by optimizing the inter-element spacing for a current soil moisture level. SMABF directivity maximization results are presented next.

SMABF Directivity Maximization Results: SMABF and virtual array's inter-element spacing which maximizes directivity in sandy and SCL soil at different soil moisture levels are shown in Fig. 10(c). Sandy soil has larger spacing

TABLE III: SMABF half wavelength inter-element spacing with change in soil moisture. All values are in $\mathrm{cm}$.

\begin{tabular}{|l|c|c|c|c|}
\hline & \multicolumn{4}{|c|}{ Volumetric Water Content (VWC) } \\
\hline \multicolumn{1}{|c|}{ Soil Type } & $\mathbf{1 0 \%}$ & $\mathbf{2 0 \%}$ & $\mathbf{3 0 \%}$ & $\mathbf{4 0 \%}$ \\
\hline Silt Loam & 30.79 & 23.72 & 20.25 & 18.03 \\
\hline Sandy & 46.83 & 39.28 & 34.62 & 31.28 \\
\hline Silty Clay Loam & 27.86 & 20.53 & 17.12 & 15.01 \\
\hline
\end{tabular}


due to low losses whereas SCL soil requires shorter interelement spacing because of higher permittivity. It can also be observed that inter-element spacing decreases with increase in soil moisture in both soils.

In Fig. 10(d), directivity of a SMABF array for inter-element spacing as a function of wavelength is shown for different steering angles in sandy soil. It can be observed that, for $d_{x} / \lambda_{s}$ values of 0.5 to 1 , higher directivity is achieved at $0^{\circ}$ and $10^{\circ}$ and it starts to fall at $30^{\circ}$ and decreases at $60^{\circ}$. Moreover, for inter-element spacing of less than $\lambda_{s} / 2$, the directivity variations between angles are low and overall directivity is lower as well. This decrease in directivity is caused by grating lobes which start to appear when $d_{x} / \lambda_{s}$ is greater than 1 or less than 0.5 .

In Fig. 10(e), effects of increase of SMABF array size on directivity are shown in SCL soil at $0^{\circ}$ steering angle. It can be observed that larger arrays have higher directivity and this effect is more pronounced for $\lambda_{s} / 2<d_{x}<\lambda_{s}$. It can be also observed that at when $d_{x} / \lambda_{s}=0.75$, by increasing the array size from $4 \times 4$ to $8 \times 8$, a 4 times increase in directivity is observed. For the $d_{x} / \lambda_{s}=1.25$, directivity of $8 \times 8$ array is significantly higher than the smaller size arrays. Hence, for a fixed inter-element spacing system, larger arrays can be used to maximize the directivity. However, when array size is increased, beamwidth of main lobe and grating lobes become narrower, therefore a small change in steering angle leads to higher variations in directivity.

\section{CONCLUSIONS}

In this paper, a soil moisture adaptive UG beamforming technique has been developed. It has been shown that when lateral wave in UG communication is exploited using SMABF, it results in improved performance of the UG communications. Soil moisture variations, change in wavelength and directivity have been identified as main challenges in UG beamforming communications. A method has been developed to find the optimal angle to focus energy in the desired direction based on soil moisture changes. SMABF is validated through simulations and empirical evaluations. Directivity analysis of the array has been presented in different soils for different soil moisture levels. SMABF outperformed both OTA fixed and soil fixed nonadaptive beamforming systems under different soil moisture levels in different soils.

\section{ACKNOWLEDGMENTS}

This work is partially supported by NSF grants NSF CNS1619285, DBI-1331895, and NSF CNS-1423379.

\section{REFERENCES}

[1] “CST Simulation Code." [Online]. Available: https://www.dropbox. com/s/sbd92aob9w11ysf/SMABF_simulations.cst?dl=0

[2] I. F. Akyildiz and E. P. Stuntebeck, "Wireless underground sensor networks: Research challenges," Ad Hoc Networks Journal (Elsevier), vol. 4, pp. 669-686, July 2006.

[3] I. F. Akyildiz, Z. Sun, and M. C. Vuran, "Signal propagation techniques for wireless underground communication networks," Physical Сотmunication Journal (Elsevier), vol. 2, no. 3, pp. 167-183, Sept. 2009.
[4] H. R. Bogena and et.al., "Potential of wireless sensor networks for measuring soil water content variability," Vadose Zone Journal, vol. 9, no. 4, pp. 1002-1013, November 2010.

[5] X. Dong and M. C. Vuran, "Environment aware connectivity for wireless underground sensor networks," in INFOCOM '13, Turin, Italy, Apr. 2013, to appear.

[6] X. Dong and M. C. Vuran, "Impacts of soil moisture on cognitive radio underground networks," in Proc. IEEE BlackSeaCom, Batumi, Georgia, July 2013.

[7] X. Dong and M. C. Vuran, "A channel model for wireless underground sensor networks using lateral waves," in Proc. of IEEE Globecom '11, Houston, TX, December 2011.

[8] X. Dong, M. C. Vuran, and S. Irmak, "Autonomous precision agriculture through integration of wireless underground sensor networks with center pivot irrigation systems," Ad Hoc Networks, vol. 11, no. 7, pp. $1975-$ 1987, 2013.

[9] A. Fenn and P. Hurst, Ultrawideband Phased Array Antenna Technology for Sensing and Communications Systems. MIT Press, 2015.

[10] L. C. Godara, "Application of antenna arrays to mobile communications. ii. beam-forming and direction-of-arrival considerations," Proceedings of the IEEE, vol. 85, no. 8, pp. 1195-1245, Aug 1997.

[11] F. Gross, Smart Antennas with MATLAB. McGraw-Hill, 2015.

[12] H. Guo and Z. Sun, "Channel and energy modeling for self-contained wireless sensor networks in oil reservoirs," IEEE Trans. Wireless Communications, vol. 13, no. 4, pp. 2258-2269, April 2014.

[13] R. Hansen, Phased Array Antennas. Wiley, 2009.

[14] R. L. Haupt, "Fitting time delay units in a large wideband corporate fed array," in 2013 IEEE Radar Conference (RadarCon13), April 2013.

[15] R. Haupt, Timed Arrays. Wiley, 2015.

[16] R. W. P. King and G. S. Smith, Antennas in Matter. The MIT Press, Jan. 1981.

[17] S. Kisseleff, I. F. Akyildiz, and W. Gerstacker, "Beamforming for magnetic induction based wireless power transfer systems with multiple receivers," in 2015 IEEE GLOBECOM, Dec 2015.

[18] S. Lakshmanan, K. Sundaresan, R. Kokku, A. Khojestepour, and S. Rangarajan, "Towards adaptive beamforming in indoor wireless networks: An experimental approach," in INFOCOM 2009, IEEE, April 2009.

[19] X. Liu and et.al., "DIRC: increasing indoor wireless capacity using directional antennas," SIGCOMM Comput. Commun. Rev., Aug. 2009.

[20] R. Mailloux, Phased Array Antenna Handbook. Artech House, 2005.

[21] N. Peplinski, F. Ulaby, and M. Dobson, "Dielectric properties of soil in the 0.3-1.3 ghz range," IEEE Transactions on Geoscience and Remote Sensing, vol. 33, no. 3, pp. 803-807, May 1995.

[22] A. Salam and M. C. Vuran, "Impacts of soil type and moisture on the capacity of multi-carrier modulation in internet of underground things," in Proc. ICCCN 2016, Hawaii, USA, Aug 2016.

[23] A. Salam and M. C. Vuran, "Smart underground antenna arrays: A soil moisture adaptive beamforming approach," Department of Computer Science and Engineering, University of Nebraska-Lincoln, Tech. Rep. TR-UNL-CSE-2017-0001, January 2017.

[24] A. Salam and M. C. Vuran, "Wireless underground channel diversity reception with multiple antennas for internet of underground things," in Proc. IEEE ICC 2017, Paris, France, May 2017.

[25] A. Salam, M. C. Vuran, and S. Irmak, "Pulses in the sand: Impulse response analysis of wireless underground channel," in Proc. INFOCOM 2016, San Francisco, USA, Apr. 2016.

[26] D. Staiman and T. Tamir, "Nature and optimisation of the ground (lateral) wave excited by submerged antennas," Proceedings of the Institution of Electrical Engineers, vol. 113, no. 8, August 1966.

[27] Z. Sun and et.al., "MISE-PIPE: Magnetic induction-based wireless sensor networks for underground pipeline monitoring," Ad Hoc Networks, vol. 9, no. 3, pp. 218-227, 2011.

[28] X. Tan, Z. Sun, and I. F. Akyildiz, "Wireless underground sensor networks: Mi-based communication systems for underground applications." IEEE Antennas and Propagation Magazine, vol. 57, no. 4, Aug 2015.

[29] M. J. Tiusanen, "Wideband antenna for underground Soil Scout transmission," IEEE Antennas and Wireless Propagation Letters, vol. 5, no. 1 , pp. 517-519, December 2006.

[30] M. Vuran, X. Dong, and D. Anthony, "Antenna for wireless underground communication," Dec. 27 2016, US Patent 9,532,118. [Online]. Available: https://www.google.com/patents/US9532118

[31] M. C. Vuran and I. F. Akyildiz, "Channel model and analysis for wireless underground sensor networks in soil medium," Physical Communication, vol. 3, no. 4, pp. 245-254, December 2010. 